\title{
PARALOGISMI E ANTINOMIE. RIFLESSIONI KANTIANE SUI CONCETTI DI SEELE E ICH DENKE
}

\author{
Davide Poggi
}

\begin{abstract}
RIASSUNTO: Il presente studio intende esaminare la presenza, nella sezione della Dialektik dedicata alla questione della Seele e ai paralogismi psicologici, di un approccio "antinomico" per ciò che concerne, in particolar modo, la questione della semplicità dell'anima. Nell'esame della tesi di Mendelssohn in merito all'incorruttibilità dell'anima, Kant mostra infatti come sia possibile assumere, accanto al criterio "estensivo", un criterio "intensivo" che porta all'affermazione della tesi della "scomponibilità" dell'anima e di una sua possibile annihilatio per remissionem. Come accade nelle antinomie, ci si trova in questo modo di fronte a due tesi in merito alla "semplicità" dell'anima in sé non-contraddittorie, ma in reciproca contrapposizione. Tali riflessioni compiute nell'edizione del 1787 (riflessioni che sembrano riprendere da vicino e perfezionare le considerazioni comparse nella Kritik del 1781 circa la semplicità del "soggetto pensante" e dell'Ich denke) trovano, a mio avviso, un'interessante "proposta di soluzione" all'interno della seconda antinomia matematica.
\end{abstract}

PAROLE CHIAVE: Psicologia razionale. Paralogismi. Ich denke. Seele. Antinomie. Quantità estensive/ intensive. Semplicità dell'anima.

\section{InTroduZione: la presenza di Moses Mendelssohn Nelle due EDIZIONI DELLA KRITIK}

Prima ancora della sezione della Dialektik interamente dedicata all'analisi della possibilità di una scienza (ossia di una conoscenza in senso stretto) della Seele, la questione della semplicità dell'anima è al centro di un breve ma interessante passaggio nelle pagine della Vorrede della Kritik del 1781. Laddove si chiede (lockianamente: POGGI, 2010) "fin dove" (wie viel) ci si possa spingere senza debordare dai limiti della certezza conoscitiva, Kant sottolinea come una risposta a tale interrogativo possa venire solo dalla "messa a processo" della ragione e del suo operato da parte della ragione stessa

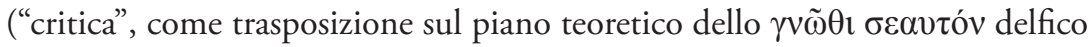
e, quindi, come "deontologia della ragion pura"):

\footnotetext{
${ }^{1}$ Dipartimento di Filosofia, Pedagogia e Psicologia. Università di Verona, Italia. davide.poggi@univr.it
} 
Ich glaube, indem ich dieses sage, in dem Gesichte des Lesers einen mit Verachtung vermischten Unwillen über dem Anscheine nach so ruhmredige und unbescheidene Ansprüche wahrzunehmen; und gleichwohl sind sie ohne Vergleichung gemäßigter, als die eines jeden Verfassers des gemeinsten Programms, der darin etwa die einfache Natur der Seele, oder die Nothwendigkeit eines ersten Weltanfanges zu beweisen vorgiebt. Denn dieser macht sich anheischig, die menschliche Erkenntnis über alle Gränzen möglicher Erfahrung hinaus zu erweitern, wovon ich demüthig gestehe, daß dieses mein Vermögen gänzlich übersteige; an dessen Statt ich es lediglich mit der Vernunft selbst und ihrem reinen Denken zu thun habe. (KrV A xiv).

Il riferimento al secondo paralogismo dell'anima è tutt'altro che velato: né ciò è a mio avviso casuale, dato che la tesi della semplicità dell'anima è considerata da Kant der Achilles, il "tallone d'Achille" di tutte le inferenze dialettiche della psicologia razionale, «[...] ein Schluß, der sogar die schärfste Prüfung und die größte Bedenklichkeit des Nachforschens auszuhalten scheint» (KrV A 351).

Ora, mentre la colpa è apertamente denunciata, affatto celato è invece il nome del colpevole, ossia, fuor di metafora, del destinatario dell'invettiva: si tratta di Moses Mendelssohn (1729-1786) che, quasi coetaneo di Kant, intrattenne con quest'ultimo un lungo rapporto epistolare e fu autore di uno dei testi più celebri della seconda metà del XVIII secolo in merito alla difesa della spiritualità dell'anima (ossia dell'a-corporeità e immortalità del principio pensante), il Phaedon oder über die Unsterblichkeit der Seele in drey Gesprächen, del 1767 (così celebre che andò incontro alla seconda edizione l'anno successivo e a una terza l'anno dopo ancora).

Perché tale autore, conosciuto e stimato da Kant (nonché, sotto certi aspetti, a lui affine: GUYER, 1991; KUEHN, 1995; TOMASONI, 2004; SASSEN, 2008), sia espressamente chiamato in causa e perché si citi esplicitamente il Phaedon, il lettore dovrà attendere la seconda edizione della Kritik (1787), nella Widerlegung des Mendelssohnschen Beweises der Beharrlichkeit der Seele (KrVB 414-B 426), inserita forse non solo in risposta alle critiche che il pensatore ebreo aveva espresso alla Kritik e al suo autore (definito der alles zermalmende) all'interno delle Morgenstunden nel 1785 (MENDELSSOHN, 1974, p.3), ma anche per ragioni puramente teoretiche, 
in ordine all'economia complessiva della proposta kantiana, così come si veniva ora definitivamente precisando ${ }^{2}$.

Resta il fatto che le tesi sostenute dal filosofo ebreo nel secondo dei tre dialoghi dell'opera di Mendelssohn erano già state oggetto delle riflessioni kantiane nel 1781, in merito all'indebita e immotivata sostanzializzazione dell'io che costituisce l'assunto di base del Phaedon in particolare e della psicologia razionale in generale (un approccio che vede le proprie origini nell'identificazione dell'ego cogito con la res cogitans, già presente nelle Meditationes cartesiane e portata avanti, in ambito tedesco, dalla metafisica di Wolff e Baumgarten, attraverso la mediazione leibniziana).

\section{Il “TALLONE D'ACHILLE”: LA POLEMICA IN MERITO ALLA SOSTANZIALIZZAZIONE DELL'ICH DENKE}

Concentriamoci sull'opera di Mendelssohn. Il quesito posto da Simmia a Socrate nel secondo dialogo del Phaedon, a proposito dello strettissimo legame tra corpo e anima per l'esercizio delle funzioni di quest'ultima, si presenta come segue:

Wo ich dich recht verstanden habe, so war den Beweis etwa folgender: Seele und Körper stehen in der genauesten Verbindung; dieser wird allmählig in seine Theile aufgelöset, jene muß entweder vernichtet werden, oder Vorstellungen haben. Durch natürliche Kräfte kann nichts zernichtet werden: daher kann unsere Seele, natürlicher Weise, niemals aufhören Begriffe zu haben. Wie aber, mein lieber Sokrates! wenn ich durch ähnliche Gründe bewiese, daß die Harmonie fortdauren müsse, wenn man auch die Leyer zerbräche, oder daß die Symmetrie eines Gebäudes noch vorhanden seyn müsse, wenn auch alle Steine von einander gerissen, und zu Staub zermalmet werden sollten? [...] Vergleichet die Leyer oder das Gebäude mit dem Körper, und die Harmonie oder Symmetrie mit der Seele: so haben wir erwiesen, daß das Saitenspiel länger dauren müsse, als die Saiten, das Ebenmaß länger, als das Gebäude. Nun ist dieses in Absicht auf die Art und Weise der Zusammensetzung andeuten: so können sie nicht länger dauren, als die Zusammensetzung selbst. Ein Gleiches läßt

\footnotetext{
${ }^{2}$ Che il rapporto tra i due pensatori fosse complesso e "chiaroscurale" lo si può evincere anche dall'ironico riferimento a Mendelssohn che Kant compie nei Prolegomena (1783): "Die alte Einrichtung der Universitätsstudien erhält noch ihren Schatten, eine einzige Akademie der Wissenschaften bewegt noch dann und wann durch ausgesetzte Preise, einen und anderen Versuch darin zu machen; aber unter gründliche Wissenschaften wird sie nicht mehr gezählt, und man mag selbst urtheilen, wie etwa ein geistreicher Mann, den man einen großen Metaphysiker nennen wollte, diesen wohlgemeinten, aber kaum von jemanden beneideten Lobspruch aufnehmen würde» (Prol, AA 04: 366).
} 
sich von der Gesundheit behaupten [...]. Reichet die Kunst des Baues hin, Pflanzen und Thieren Leben und Gesundheit zu geben, so kann eine höhere Kunst vielleicht dem Thiere Empfindung, und dem Menschen Vernunft verleihen. Wir blödsinnigen begreifen jenes so wenig, als dieses. [...] Wollen wir der Allmacht oder der Weisheit des Schöpfers Grenzen setzen? (MENDELSSOHN, 1972, II, p.81-82).

Il che, da un esame comparato del testo di Mendelssohn e di quello platonico, si configura come una rilettura del problema già proposto in quest'ultimo alla luce degli sviluppi materialistici che la thinking matter question, lasciata aperta da Locke all'interno dell'Essay concerning Humane Understanding $\left(1690^{1}\right)$, aveva ricevuto nel corso del Settecento (LOCKE, 1975, IV, 3, 6, p.539). Nel richiamo alla tesi secondo la quale l'ignoranza umana circa l'unione di anima e corpo nell'unico ente "uomo" non deve essere applicata all'onnipotenza del Creatore, dato che ne costituirebbe una illecita limitazione, non si può infatti non avvertire l'eco dell'esaltazione dell'insondabilità dell'Omnipotency contenuta nella Second Reply di Locke a Edward Stillingfleet ${ }^{3}$. La risposta di Socrate-Mendelssohn verte proprio sul concetto di semplicità e consiste nell'esame della possibilità di un "rapporto circolare" tra un fatto e le condizioni che lo determinano: se il pensiero, come facoltà di pensare, è richiesto come conditio formale per poter parlare di armonia di partes extra partes (la cui effettiva disposizione ne è la conditio materiale), esso non può essere al contempo l'effetto o l'epifenomeno di tale disposizione di parti e delle loro proprietà, ma qualcosa di originario rispetto ad esse:

Das denkende Vermögen, und dieses allein in der ganzen Natur, ist fähig, durch eine innerliche Thätigkeit Vergleichungen, Verbindungen und Gegeneinanderhaltungen wirklich zu machen: daher der Ursprung alles Zusammengesetzten, der Zahlen, Größen, Symmetrie, Harmonie u. s. w. in so weit sie ein Vergleichen und Gegeneinanderhalten erfordern, einzig und allein in dem denkenden Vermögen zu suchen seyn muß. Und da dieses zugegeben wird, so kann ja dieses Denkungsvermögen selbst [...] unmöglich aus diesen ihren eigenen Verrichtungen entspringen, unmöglich in einem Verhältniß, Harmonie, Symmetrie, unmöglich in einem Ganzen bestehen, das aus außereinander seyenden Theilen zusammengesetzt ist: denn alle

\footnotetext{
${ }^{3}$ "I know nobody, before Des Cartes, that ever pretended to show that there was any contradiction in it. So that at worst, my not being able to see in matter any such incapacity, as makes it impossible for omnipotency to bestow on it a faculty of thinking, makes me opposite only to the Cartesians. For as far as I have seen or heard, the fathers of the christian church never pretended to demonstrate that matter was incapable to receive a power of sensation, perception and thinking, from the hand of the omnipotent creator» (LOCKE, 1997, p.469).
} 
diese Dinge setzen die Wirkungen und Verrichtungen des denkenden Wesens voraus, und können nicht anders, als durch dieselben, wirklich werden. [...] So kann ich den Ursprung dieses Vorstellungsvermögens selbst nicht in ein Ganzes setzen, das aus solchen auseinanderseyenden Theilen bestehet, ohne eine Sache durch ihre eigenen Verrichtungen entstehen zu lassen. (MENDELSSOHN, 1972, II, p.92-93).

Tutto questo non è però ancora sufficiente per garantire la semplicità dell'anima: affinché la dimostrazione sia completa, osserva Socrate, occorre assumere l'ipotesi per cui l'anima sarebbe l'effetto della composizione e, quindi, delle forze dei componenti in gioco. Due sono le possibilità: i componenti e il risultato finale possono cioè essere qualitativamente diversi, oppure simili. Nel primo caso (situazione analoga a quella delle sillabe e della parola che esse vanno a comporre), è necessario riconoscere come originaria rispetto al composto l'esistenza di un essere pensante, in quanto si tratterebbe dell'unico essere capace di cogliere il molteplice in un'unità e rappresentare i componenti diversamente dal modo in cui essi sono singolarmente e separatamente ${ }^{4}$. Il secondo caso è invece quello dei contenuti psichici (leibnizianamente, Vorstellungen) che l'esperienza introspettiva mostra susseguirsi incessantemente in noi. Affinché si possa parlare di "pensiero" in senso stretto occorre che questi ultimi mettano capo a un'unica sostanza pensante-rappresentativa:

\footnotetext{
${ }^{4}$ "Wir wollen also diesen Fall setzen, fuhr Sokrates fort, und annehmen, unsere Seele sey eine Wirksamkeit des Zusammengesetzten. Wir haben gefunden, daß alle Wirksamkeiten des Zusammengsetzten aus den Kräften der Bestandtheile fließen müssen: werden also nach unserer Voraussetzung, die Bestandtheile unsers Körpers nicht Kräfte haben müssen, aus denen im Zusammengesetzten das Vermögen zu denken resultiret? [...] Aber die Kräfte dieser Bestandtheile, von welcher Natur und Beschaffenheit wollen wir sie annehmen? Sollen sie der denkenden Thätigkeit ähnlich oder unähnlich seyn? [...] Eine einzelne Sylbe, sprach S o kr at es, hat mit der ganzen Rede dieses gemein, daß sie vernehmlich ist; aber die ganze Rede hat einen Verstand, die Sylbe keinen: Nicht? [...] Indem also nur jede Sylbe ein zwar vernehmliches, aber verstandleeres Gefühl erregt, so entspringet aus ihrem Inbegriffe dennoch ein verständiger Sinn, der auf unsere Seele wirkt. Allhier resultiret die Wirksamkeit des Ganzen aus den Kräften der Theile, die ihnen unähnlich sind. [...] Wenn aus einfachen Kräften eine von ihnen verschiedene Kraft im Zusammengesetzten entspringen soll, wo kann diese Verschiedenheit anzutreffen seyn? Außer dem denkenden Wesen sind die Kräfte des Ganzen nichts anders, als die einzelnen Kräfte der einfachen Bestandtheile, wie sie sich durch Wirkungen und Gegenwirkungen einander abändern, und einschränken. Von dieser Seite findet also die Unähnlichkeit nicht statt, und wir müssen abermals unsere Zuflucht zu dem denkenden Wesen nehmen, das die Kräfte in Verbindung und zusammengenommen sich anders vorstellet, als sie dieselben einzeln und ohne Verbindung denken würde» (MENDELSSOHN, 1972, II, p.94-95).
} 
Wir würden weder uns erinnern, noch überlegen, noch vergleichen, noch denken können, ja wir würden nicht einmal die Person seyn, die wir vor einem Augenblick gewesen, wenn unsere Begriffe unter vielen vertheilet und nicht irgend wo zusammen in ihrer genauesten Verbindung anzutreffen wären. [...] Es giebt also in unserm Körper wenigstens eine einzige Substanz, die nicht ausgedehnt, nicht zusammengesetzt, sondern einfach ist, eine Vorstellungskraft hat, und alle unsere Begriffe, Begierden und Neigungen in sich vereiniget. Was hindert uns, diese Substanz Seele zu nennen? (MENDELSSOHN, 1972, II, p.96-97).

Come precedentemente anticipato, quanto proposto da Kant nella Kritik des zweiten Paralogisms della prima edizione della Kritik costituisce a mio avviso un dialogo indiretto e soltanto "ideale" con Mendelssohn: quando il filosofo di Königsberg passa a spiegare il ragionamento che sta alla base del "sillogismo psicologico della semplicità" propone infatti un esempio, quello delle singole parole di un verso e del verso compiuto dotato di senso ${ }^{5}$, analogo a quello addotto nel Phaedon, ossia all'esempio delle sillabe e della parola/ proposizione che ne deriva (MENDELSSOHN, 1972, II, p.94). Si tratta, del resto, della rielaborazione di un'argomentazione tipica della psicologia razionale che Kant ben conosceva. La ritroviamo infatti nelle lezioni di psicologia ${ }^{6} \mathrm{da}$

${ }^{5}$ "Eine jede zusammengeseßte Substanz ist ein Aggreg[r]at vieler, und die Handlung eines Zusammengeseßten oder das, was ihm als einem solchen inhärirt, ist ein Aggregat vieler Handlungen oder Accidenzen, welche unter der Menge der Substanzen vertheilt sind. Nun ist zwar eine Wirkung, die aus der Concurrenz vieler handelnden Substanzen entspringt, möglich, wenn diese Wirkung bloß äußerlich ist (wie z. B. die Bewegung eines Körpers die vereinigte Bewegung aller seiner Theile ist). Allein mit Gedanken, als innerlich zu einem denkenden Wesen gehörigen Accidenzen, ist es anders beschaffen. Denn seßet, das Zusammengeseßte dächte: so würde ein jeder Theil desselben einen Theil des Gedanken, alle aber zusammengenommen allererst den ganzen Gedanken enthalten. Nun ist dieses aber widersprechend. Denn weil die Vorstellungen, die unter verschiedenen Wesen vertheilt sind, (z. B. die einzelne Wörter eines Verses) niemals einen ganzen Gedanken (einen Vers) ausmachen: so kann der Gedanke nicht einem Zusammengeseßten als einem solchen inhäriren. Er ist also nur in einer Substanz möglich, die nicht ein Aggregat von vielen, mithin schlechterdings einfach ist» ( $\mathrm{KrV}$ A 351-352).

${ }^{6}$ «Die Seele ist einfach, d. h. das Ich bedeutet einen einfachen Begriff. Viele Wesen können nicht zusammengenommen ein Ich ausmachen. Wenn ich sage: Ich denke; so drücke ich nicht Vorstellungen aus, die unter viele Wesen vertheilt sind, sondern ich drücke eine Vorstellung aus, die bei Einem Subjecte statt findet. Denn alle Gedanken können nur einfach oder zusammen gesetzt seyn. Ein und eben derselbe einfache Gedanke kann nur in einem einfachen Subjecte statt finden. Denn wenn die Theile der Vorstellungen sollten eingetheilt seyn unter viele Subjecte; so hätte jedes Subject nur einen Theil der Vorstellung; mithin hätte kein einziges Subject ganz sey; so müssen auch alle Theile der Vorstellung in dem einen Subjecte seyn. Denn wenn sie nicht zusammen in dem einen Subjecte verbunden sind; so ist die Vorstellung nicht ganz. Z. E. wenn der Ausspruch: quidquid agis etc. unter viele Subjecte sollte vertheilt werden, so daß jedes einen Theil hätte; wenn nämlich dem einen das Wort quidquid, dem andern agis ins Ohr gesagt würde, so daß teine den ganzen Spruch hörte; so könnte man nicht sagen: der ganze Gedanke sey in den vielen Köpfen zusammen, so daß 
lui tenute presso l'università di Königsberg verso la fine degli anni settanta del Settecento (durante il "ventennio gestazionale" che separò la Dissertatio del 1770 e la prima edizione della Kritik), sulla scorta del testo su cui tali lezioni erano calibrate, ossia la Metaphysica di Baumgarten (BAUMGARTEN, 17797 $\$ 742$, p. 293-295).

Ora, in cosa consiste l'errore che trasformerebbe in un paralogismo l'argomento per cui il pensiero richiede l'unità impartibile del soggetto pensante e quindi la sua assoluta semplicità? La risposta kantiana consiste nella ripresa e nel perfezionamento della critica al primo paralogismo, giacché ruota attorno all'insensibile passaggio dall'Ich denke, inteso sul piano meramente logico come conditio trascendentale di ogni esperienza possibile (ossia come l'unità sintetica originaria a priori per la "conoscenza di oggetti", a prescindere dai contenuti empiricamente dati e dall'appercezione empirica che accompagna le singole rappresentazioni), all' $I c h$ denke come il concetto di un essere pensante in generale, ossia sul piano noumenico, di cui si ritiene erroneamente di poter dedurre il carattere di unità assoluta.

Si ha cioè un salto da una semplicità formale priva di ogni determinazione a una semplicità che, in quanto "assoluta", non può rientrare nell'ambito del conoscibile (per l'uomo) e non può neppure essere dedotta dalla natura del pensiero. In questo risiede la differenza rispetto alla semplicità dell'appercezione pura trascendentale, la cui unità qualitativa o semplicità sovracategoriale, ossia non riconducibile alle sintesi cognitive di cui è condizione ${ }^{7}$, non è una "conoscenza" dell'io-soggetto.

Una sottile (ma fondamentale) differenza che consente a mio avviso di risolvere l'apparente contraddizione tra quanto osservato da Kant nel 1781, là dove affermava che la proposizione «Ein Gedanke kann nur die Wirkung der absoluten Einheit des denkenden Wesens sein» (KrV A 353) non può essere ritenuta "analitica", e quanto scrive nel 1787, ossia che il «Grundsatz, der notwendigen Einheit der Apperception ist [...] identisch, mithin ein analytischer Satz» (KrV B 135) - per quanto sia necessaria una sintesi come condizione del pensamento di tale identico Ich denke.

jeder einen Theil von dem Gedanken hätte; sondern der Gedanke ist gar nicht, indem nur jeder den Gedanken von einem Worte hat, aber nicht einen Theil der ganzen Vorstellung» (V-Met-L1/Pölitz, AA 28: 266-267).

${ }^{7}$ Si tratta di un ragionamento proposto nella Deduzione trascendentale della seconda edizione della Kritik che, giustamente, Sassen osserva essere affine a quello proposto da Mendelssohn sull'irriducibilità del soggetto pensante all'armonia di cui è conditio formale (SASSEN, 2008, p.227-232). 
Né il ragionamento compiuto nella Kritik des zweiten Paralogisms si arresta qui, poiché la questione della semplicità dell'anima, come Kant stesso riconosce, è significativa nella misura in cui tale semplicità costituisce la cifra che distingue l'anima dal corpo (con tutto ciò che ne è corollario per ciò che concerne la possibilità di una morte "naturale" per s-composizione): la seconda parte dello smascheramento del "tallone d'Achille" è quindi incentrata sul fatto che la contrapposizione anima-corpo, così come è stata proposta dalla psicologia razionale, si fonda sull'assunzione della semplicità (dell'anima) e della composizione (della materia) come caratteri della cosa in sé, e non solo come determinazioni, rispettivamente, delle rappresentazioni del senso interno e del senso esterno (come invece dovrebbe essere). Scrive Kant:

Wäre Materie ein Ding an sich selbst, so würde sie als ein zusammengesetztes Wesen von der Seele als einem einfachen sich ganz und gar unterscheiden. Nun ist sie aber blos äußere Erscheinung, deren Substratum durch gar keine anzugebende Prädikate erkannt wird; mithin kann ich von diesem wohl annehmen, daß es an sich einfach sei, ob es zwar in der Art, wie es unsere Sinne afficirt, in uns die Anschauung des Ausgedehnten und mithin Zusammengesetzten hervorbringt, und daß also der Substanz, der in Ansehung unseres äußeren Sinnes Ausdehnung zukommt, an sich selbst Gedanken beiwohnen, die durch ihren eigenen inneren Sinn mit Bewußtsein vorgestellt werden können. Auf solche Weise würde eben dasselbe, was in einer Beziehung körperlich heißt, in einer andern zugleich ein denkend Wesen sein, dessen Gedanken wir zwar nicht, aber doch die Zeichen derselben in der Erscheinung anschauen können. (KrV A 359).

Proprio la corretta comprensione del significato dei concetti di "soggetto pensante", "io" e di "materia" (che lascia impregiudicata la natura dell'intelligibile che sta alla base di entrambi), lungi dal risolvere in maniera definitiva la questione della contrapposizione anima-corpo incentrata sulla semplicità, la lascia aperta, generando una situazione di ambigua indecidibilità. Chi può infatti garantire che il noumenon che si manifesta a noi attraverso il senso esterno (assumendo quindi le qualità fenomenologiche dell'estensione, della scomponibilità, etc.) non sia invece caratterizzato, al pari del soggetto in quanto si conosce come pensante attraverso il senso interno, dalla "semplicità" e dall'"in estensione"? In questo caso, non si avrebbe contrapposizione assoluta, ma solo relativa e, per giunta, relativa a qualcosa che non potremmo mai falsificare. 
Siamo cioè di fronte a uno slittamento del discorso dal contesto dei paralogismi a quello delle antinomie, cosa che Kant conferma proponendo una più concisa versione dei sopraccitati ragionamenti ( $\mathrm{D}$ a es oben ausführlicher erwogen ist»: KrV A 443/B 471) nella Anmerkung zur Antithesis dello Zweiter Widerstreit:

Es bringt also nur das Selbstbewußtsein es so mit sich, daß, weil das Subject, welches denkt, zugleich sein eigenes Object ist, es sich selber nicht theilen kann (obgleich die ihm inhärirende Bestimmungen) [...]. Nichts destoweniger, wenn dieses Subject äußerlich, als ein Gegenstand der Anschauung, betrachtet wird, so würde es doch wohl Zusammensetzung in der Erscheinung an sich zeigen. So muß es aber jederzeit betrachtet werden, wenn man wissen will, ob in ihm ein Mannigfaltiges a u $ß$ e r h a lb einander sei, oder nicht. (KrV A 443/B 471).

Si tratta di un passo interessante, non solo per il contenuto (che, ancora una volta, si pone in continuità con alcune riflessioni compiute dal pensatore di Königsberg nelle lezioni di psicologia presso l'Albertina) ${ }^{8}$, ma per quello che rappresenta nell'architettura generale dell'opera: il fatto che Kant lo mantenga nella seconda edizione della Kritik (lo si ritroverà in B 471), laddove invece espungerà la corrispondente versione "estesa" nel contesto della rivisitazione

\footnotetext{
${ }^{8}$ «Alle Objecte des äußern Sinnes sind materiell, und dadurch werde ich die Gegenstände des äußern Sinnes gewahr, wenn sie durch Undurchdringlichkeit im Raum gegenwärtig sind. Der Seele aber bin ich mir durch den innern und nicht durch den äußern Sinn bewußt; also sehe ich ein, daß mir die Seele als ein Object des innern Sinnes gegeben ist. Ferner so sehen wir, daß alle Handlungen der Seele, das Denken, Wollen usw. Keine Objecte des äußern Sinnes sind. Ein denkendes Wesen, als ein solches, kann gar kein Object des äußeren Sinnes seyn; wir können weder das Denken, noch das Wollen, noch das Vermögen der Lust und Unlust durch den äußern Sinn wahrnehmen; und wir können uns nicht vorstellen, wie die Seele als ein denkendes Wesen ein Object des äußeren Sinnes seyn sollte; allein da sie das nicht ist, so ist sie auch nicht materiell. [...] Wir haben aber schon dargethan, daß die Seele eine Substanz, und dann daß sie eine einfache Substanz sey. Daraus glaubte Wolff schon die Immaterialität zu beweisen; allein das ist falsch; aus der Simplicität folgt noch nicht die Immaterialität; denn der kleinste Theil eines Körpers ist doch wirklich etwas materielles und ein Gegenstand des äußern Sinnes. Ob er gleich kein wirklicher Gegenstand der äußern Sinne ist; so kann er doch durch die Zusammensetzung von vielen solchen kleinen Theilchen ein wirklicher Gegenstand des äußeren Sinnes werden. [...] Woraus kann ein Philosoph die Immaterialität der Seele beweisen, und wie weit kann er gehen? Er kann von nichts anders die Gedanken des innern Sinnes ausdrückt. Also liegt in dem Begriffe vom Ich die Immaterialität. - Wir können a priori die Immaterialität der Seele nicht beweisen, sondern nur so viel: $\mathrm{da} ß$ alle Eigenschaften und Handlungen der Seele sich nicht aus der Materialität erkennen lassen. Allein diese Eigenschaften beweisen noch nicht, daß unsere Seele nichts Aeußeres haben sollte; sondern nur so viel, daß ich die Materialität nicht als einen Erklärungsgrund der Handlungen annehmen kann. Ich schließe also nur die Materialität aus» (V-Met-L1/Pölitz, AA 28: 271-273).
} 
del secondo paralogismo, è indice dell'intenzione di "essenzializzare" la confutazione della psicologia razionale e, nello specifico, di collegare la questione psicologica al peculiare modus argumentandi delle antinomie.

Ora, desidero concentrarmi proprio sulla seconda edizione della Kritik, al fine di far emergere la presenza di alcuni interessanti punti di contatto tra l'esame kantiano del concetto di Seele e di Ich denke compiuto nella sezione dedicata ai paralogismi e le riflessioni sviluppate a riguardo delle antinomie (in particolar modo la seconda).

\section{La CONFutazione DElla prova di MENDElSSOHN: LO SCENARIO DI UN'ANTINOMIA PSICOLOGICA}

Nel 1787 la questione della semplicità del soggetto pensante-Seele è affrontata da Kant con l'intenzione di puntare al cuore della presunta scienza quale è la psicologia razionale, denunciando l'indebita applicazione della categoria della sostanza all'"io penso" e quindi la confusione tra la semplicità logico-formale dell'io penso ("der ärmsten Vorstellungen unter allen»: $\mathrm{KrV}$ B 408) e la semplicità della sostanza pensante. Il "tallone d'Achille" è ancora oggetto di confutazione, ma il suo ruolo è alquanto de-potenziato:

Daß das Ich der Apperception [...] in jedem Denken ein Singular sei, der nicht in eine Vielheit der Subjecte aufgelöset werden kann, mithin ein logisch einfaches Subject bezeichne, liegt schon im Begriffe des Denkens, ist folglich ein analytischer Satz; aber das bedeutet nicht, daß das denkende Ich eine einfache $S$ ubstan z sei, welches ein synthetischer Satz sein würde. (KrV B 407-408).

Se ci si limitasse a questa critica, sembrerebbe lecito affermare che nulla di realmente nuovo è stato aggiunto rispetto all'edizione del 1781 e che il filosofo ha semplicemente operato un labor limae, intervenendo sulle modalità espositive e non tanto sul piano concettuale.

Che questa sia tuttavia solo una semplice impressione lo si evince dal prosieguo del testo della Kritik, ossia dalla Widerlegung dell'argomento mendellsohniano circa la permanenza dell'anima e, in ultima analisi, circa la sua semplicità. L'argomento del "divenire" come graduale continuum di mutamenti esposto nel primo dialogo del Phaedon per bocca di Socrate si presenta come segue: 
Die Sätze [...] deren Richtigkeit wir nicht mehr in Zweifel ziehen, sind diese:

1) $\mathrm{Zu}$ einer jeden natürlichen Veränderung wird dreyerley erfodert: I) Ein Zustand eines veränderlichen Dinges, der aufhören, II) ein anderer, der seine Stelle vertreten soll, und III) die mittlern Zustände, oder der Uebergang, damit die Veränderung nicht plötzlich, sondern allmählig geschehe.

2) Was veränderlich ist, bleibet keinen Augenblick, ohne wirklich verändert zu werden.

3) Die Folge der Zeit gehet in einem fort, und es giebt keine zween Augenblicke, die sich einander die nächsten sind.

4) Die Folge der Veränderungen kömmt mit der Folge der Zeit überein, und ist ebenfalls so stätig, so unzertrennlich, daß man keine Zustände angeben kann, die sich einander die nächsten wären, oder zwischen welchen nicht ein Uebergang Statt finden sollte. (MENDELSSOHN, 1972, I, p.65-66) 9 .

Ciò posto, una "morte naturale" come passaggio dal Seyn al Nichtseyn non è affatto possibile, essendo sempre individuabile una fase intermedia del mutamento ${ }^{10}$. Così, se per il corpo non si può parlare di morte in senso assoluto, ma piuttosto di una fase in cui ciò che è ritenuto morto si sottrae in effetti alla nostra sensibilità, ossia a una conoscenza distinta, per l'anima (in un ragionamento analogico fondato sull'ipotesi provvisoria della semplicità dell'anima) si aprono invece due prospettive: da una parte la morte per immediato e improvviso "annientamento" (in maniera naturale oppure durch ein Wunderwerk, caso, quest'ultimo, ritenuto inammissibile: MENDELSSOHN, 1972, I, p.70), dall'altra la morte per progressiva perdita/ svanimento "[...] della forza e dell'efficacia interiore", "wie sich die Theile der Maschine trennen» (MENDELSSOHN, 1972, I, p.71). Si tratta di un dettaglio, questo della corrispondenza sincronica tra il piano del corpo e quello dell'anima, che non va a mio avviso trascurato, dato che l'argomento della morte dell'anima per "illanguidimento" viene presentato esclusivamente in quest'ottica ${ }^{11}$. In entrambi $\mathrm{i}$ casi, osserva Socrate, sarebbe comunque impossibile una morte naturale, giacché:

${ }^{9}$ L'argomento mendelssohniano non è ripreso da Platone, bensì dal pensiero leibniziano-boscovichiano (MARTINELLI, 2002, p.93-94; TOMASONI, 2011, p.147).

${ }_{10}$ "Zwischen Seyn und Nichtseyn ist eine entsetzliche Kluft, die von der allmählig wirkenden Natur der Dinge nicht übersprungen werden kann» (MENDELLSOHN, 1972, I, p.70).

11 "[Sokrates] Die eine Todesart, die plötzliche Zernichtung, schreckt uns also nicht mehr, fuhr Sokrates fort; denn sie ist in der Natur unmöglich. Doch überlegt auch folgendes, meine Freunde. 
Der letzte Schritt, man mag ihn noch so weit hinaus schieben, wäre immer noch vom Daseyn zum Nichts ein Sprung, der weder in dem Wesen eines einzelnen Dinges, noch in dem ganzen Zusammenhange gegründet seyn kann. Sie wird also fort dauren, ewig vorhanden seyn. Soll sie vorhanden seyn, so muß sie wirken und leiden; soll sie wirken und leiden, so muß sie Begriffe haben. (MENDELSSOHN, 1972, I, p.73).

Ora, come osserva Sassen, lo scopo di Kant nella confutazione della prova mendelssohniana, non pare essere quello di confutare Mendelssohn sul suo stesso piano, ossia seguendo l'ordine di ragionamenti da lui proposti e guardando ai presupposti sui quali si fondano le tesi del filosofo ebreo (vale a dire la concezione del divenire come ininterrotto susseguirsi di stati e l'impossibilità del salto dall'essere al non essere: SASSEN, 2008, p.220). La citazione compiuta dal filosofo di Königsberg non può tuttavia dirsi rigorosa, bensì sommaria e solo parzialmente corrispondente con quanto si trova nel Phaedon, giacché lascia pensare che il nervus probandi del discorso mendelssohniano consista nell'impossibilità di una graduale trasformazione in nulla, a causa della semplicità dell'anima in relazione a ciò che costituisce la sua esistenza. Egli scrive:

Dieser scharfsinnige Philosoph merkte bald in dem gewöhnlichen Argumente, dadurch bewiesen werden soll, daß die Seele (wenn man einräumt, sie sei ein einfaches Wesen) nicht durch Zertheilung zu sein aufhören könne, einen Mangel der Zulänglichkeit zu der Absicht, ihr die nothwendige Fortdauer zu sichern, indem man noch ein Aufhören ihres Daseins durch Verschwinden annehmen könnte. In seinem Phädon suchte

Gesetzt sie wäre nicht unmöglich, so ist die Frage: wann? [...] Vermuthlich zu der Zeit, da der Körper ihrer nicht mehr bedarf, in dem Augenblicke des Todes? - [Cebes] Allem Ansehen nach. - Nun haben wir aber gesehen, daß es keinen bestimmten Augenblick giebt, da man sagen kann, itzt stirbt das Thier. Die Aufösung der thierischen Machine hat schon lange vorher ihren Anfang genommen, ehe noch ihre Wirkungen sichtbar geworden sind; denn es fehlet niemals an solchen thierischen Bewegungen, die der Erhaltung des Ganzen zuwider sind; nur daß sie nach und nach zunehmen, bis endlich alle Bewegungen der Theile nicht mehr zu einem einzigen Endzwecke harmoniren, sondern eine jede ihren besondern Endzweck angenommen hat: und alsdann ist die Maschine aufgelöset. Dieses geschiehet so allmählig, in einer so stetigen Ordnung, daß jeder Zustand eine gemeinschaftliche Grenze des vorhergehenden und nachfolgenden Zustandes, eine Wirkung des vorhergehenden und eine Ursache des nachfolgenden Zustandes zu nennen ist. [...] Wenn also der Tod des Körpers auch der Tod der Seele seyn soll: so muß es auch keinen Augenblick geben, da man sagen kann, itzt verschwindet die Seele; sondern nach und nach, wie die Bewegungen in den Theilen der Maschine aufhören zu einem einzigen Endzwecke zu harmoniren, muß die Seele auch an Kraft und innerer Wirksamkeit abnehmen. [...] Wir haben also nur noch dieses zu untersuchen, ob die inneren Kräfte der Seele nicht so allmählig vergehen können, wie sich die Theile der Maschine trennen» (MENDELSSOHN, 1972, I, p.70-71). 
er nun diese Vergänglichkeit, welche eine wahre Vernichtung sein würde, von ihr dadurch abzuhalten, daß er sich zu beweisen getraute: ein einfaches Wesen könne gar nicht aufhören zu sein, weil, da es gar nicht vermindert werden und also nach und nach etwas an seinem Dasein verlieren und so allmählig in Nichts verwandelt werden könne (indem es keine Theile, also auch keine Vielheit in sich habe), zwischen einem Augenblicke, darin es ist, und dem andern, darin es nicht mehr ist, gar keine Zeit angetroffen werden würde, welches unmöglich ist. (KrV B 413-414).

L'ipotesi formulata in merito da Sassen, ossia che Kant abbia citato il Phaedon senza avere il testo "a portata di mano", basandosi cioè solamente sui ricordi di una lettura tutt'altro che recente dell'opera di Mendelssohn (SASSEN, 2008, p.219-220), se è in sé e per sé certamente plausibile, mal si accorda tuttavia con il fatto, evidenziato da Martinelli (2002, p.102) e da Wuerth (2010, p.221-234), che il filosofo di Königsberg mostra di essersi concentrato in altre occasioni sulla questione della semplicità dell'anima e sulla possibilità della progressiva remissione delle sue facoltà in termini assai vicini a quelli poi esposti nella seconda edizione della Kritik, come ad esempio nel 1786, all'interno dei Metaphysische Anfangsgründe der Naturwissenschaft (MAN, AA 04: 542) ${ }^{12}$. Tale impasse interpretativa può a mio avviso trovare una soluzione non appena si porti l'attenzione sulle modalità in cui Kant presenta gli argomenti mendelssohniani e sullo scopo che egli si era prefisso. Esaminiamo il prosieguo della Widerlegung:

Allein er bedachte nicht, daß, wenn wir gleich der Seele diese einfache Natur einräumen, da sie nämlich kein Mannigfaltiges außer einander, mithin keine extensive Größe enthält, man ihr doch, so wenig wie irgend einem Existirenden, intensive Größe, d. i. einen Grad der Realität in Ansehung aller ihrer Vermögen, ja überhaupt alles dessen, was das Dasein ausmacht, ableugnen könne, welcher durch alle unendlich viele kleinere Grade abnehmen und so die vorgebliche Substanz (das Ding, dessen Beharrlichkeit nicht sonst schon fest steht), obgleich nicht durch Zertheilung, doch durch allmählige Nachlassung (remissio) ihrer Kräfte, (mithin durch Elanguescenz, wenn es mir erlaubt ist, mich dieses Ausdrucks zu bedienen) in Nichts verwandelt werden könne. (KrV B 414).

\footnotetext{
${ }^{12}$ Wuerth, in particolar modo, riporta l'elenco dei testi in cui Kant affronta il tema della semplicità dell'anima in relazione alla sua corruttibilità successivamente al 1781 (ometterò, per ovvie ragioni, la seconda edizione della Kritik): V-Met/Mron, AA 29: 905; V-Met-K2/Heinze, AA 28: 763; V-MetK3-Vigilantius, AA 29: 1037.
} 
Kant sembra qui esporsi all'accusa di aver compiuto un'ignoratio elenchi, poiché presenta l'argomento contro l'estinzione come se essa fosse l'unica prova proposta dal pensatore ebreo e mostra di considerare il criterio intensivo come in-edito (scrive infatti «Allein er bedachte nicht, daß, wenn [...]»), quando invece Mendelssohn parla effettivamente di una "perdita di forza e vigore interno", come è stato sottolineato (PARDEY, 1999, p.274) ${ }^{13}$. ̀̀ a mio avviso alquanto plausibile che il filosofo di Königsberg sia stato indotto a ciò dal fatto che nel Phaedon l'illanguidimento delle facoltà dell'anima è stato proposto in un rapporto di stretta analogia con la dissoluzione corporea e, conseguentemente, il dis-armonizzarsi delle varie facoltà dell'unica Fundamentalkraft dell'anima (la leibniziana forza rappresentativa) descritto in termini presque spaziali. Una caduta nella concezione della semplicità come non-composizione (anche di ciò che, per sua natura, è inesteso, come è appunto l'anima) che è, a mio avviso, presupposta da Kant ${ }^{14}$ e addirittura accentuata nell'esposizione delle tesi del pensatore ebreo, tanto da farne quasi il cuore dei ragionamenti di quest'ultimo. A dire il vero, laddove la prova del Phaedon non si fondava tanto su tale non-composizione, quanto piuttosto sull'impossibilità del passaggio dall'essere al non essere per via naturale, poiché tale passaggio richiede la violazione dell'assunto per cui il "divenire naturale" è un continuum ininterrotto.

Ora, se tutto ciò è utile per comprendere la specificità della presentazione kantiana delle prove mendelssohniane, quello che è estremamente importante ai fini del presente discorso (e ci indica la via per un'adeguata interpretazione della Widerlegung) è il fatto che Kant intende qui proporre un argomento che intende avvalersi di un preciso cambio di prospettiva: il ragionamento kantiano è cioè interamente fondato sull'assunzione del criterio della quantità intensiva e l'accentuazione di cui si parlava precedentemente è funzionale alla contrapposizione estensivo/intensivo. Come era emerso nelle Anticipationen

${ }^{13}$ Analogamente a Pardey, ritengo che la concezione mendelssohniana della "forza intensiva" sia da individuare nel primo dei tre dialoghi del Phaedon (e non nel terzo, come invece è suggerito in: FALKENSTEIN, 1998, p.588). Anche Martinelli riconosce come in Mendelssohn si trovi una trattazione della "forza intensiva" (non solo all'interno del Phaedon, ma sin dalla Preisschrift del 1763, per quanto in un'accezione ritenuta differente da quella adottata da Kant: MARTINELLI, 2002, p.107-110)

${ }^{14}$ In tal modo, è a mio avviso possibile condividere la tesi di Martinelli per cui la Widerlegung kantiana non si fonderebbe su un mero misunderstanding degli argomenti di Mendelssohn (MARTINELLI, 2002, p. 94, 102, 124); Martinelli si pone in aperto contrasto con una linea interpretativa della Widerlegung kantiana che ne evidenziava le inesattezze e la non corrispondenza con le tesi del Phaedon (VIEILLARD-BARON, 1974; PARDEY, 1999). 
der Wahrnehmung (dove compare la prima occorrenza dell'aggettivo intensive in entrambe le edizioni della Kritik: KrV A 166/B 208), la quantità intensiva riguarda primariamente das Reale der Empfindung ${ }^{15}$, la presenza di un'affezione del soggetto e, tra le qualità, è l'unica che può essere conosciuta a priori, giacché è sempre possibile stabilire che, data una qualsivoglia percezione (sensazione accompagnata da coscienza), essa ha un grado effettivo appreso istantaneamente (l'esserci della modificazione) e tale grado può decrescere in maniera infinitesimale fino a un "grado zero" (l'assenza della sensazione). Tale quantità intensiva riguarda sia gli Objekten der Wahrnehmung (perché ad essi deve essere attribuito un certo grado di influsso sui sensi del soggetto) sia la Seele (ossia il soggetto in quanto oggetto del senso interno) con le sue facoltà e in primis la coscienza di sé, come Kant già aveva osservato nei Metaphysische Anfangsgründe (MAN, AA 04: 542) (il che, analogamente al discorso sulla semplicità dell'anima qua non-composta, si inscrive alla perfezione in un contesto di psicologia razionale).

Ciò significa rendere complessa, divisibile e passibile di estinzione la sostanza dell'anima, lasciando impregiudicata la sua "semplicità-noncomposizionale", poiché, anche riconoscendo che la Seele non è oggetto del senso esterno e quindi non possiede una quantità estensiva (non è costituita da partes extra partes), può tuttavia possedere una quantità intensiva, come osserva Kant nella seguente nota della Widerlegung:

Obzwar die Theilbarkeit ein Zusammengesetztes voraussetzt, so erfordert sie doch nicht nothwendig ein Zusammengesetztes von Substanzen, sondern bloß von Graden (der mancherlei Vermögen) einer und derselben Substanz. (KrV B 416).

Così, nonostante Kant, all'inizio del capitolo sulle antinomie della ragion pura, sottolinei che:

Es ist $[\ldots]$ merkwürdig, daß der transcendentale Paralogism einen bloß einseitigen Schein in Ansehung der Idee von dem Subjecte unseres Denkens bewirkte, und zur Behauptung des Gegentheils sich nicht der mindeste Schein aus Vernunftbegriffen vorfinden will. (KrV B 433).

si ha una situazione a mio avviso del tutto analoga alle antinomie. Al paralogismo si aggiunge infatti un'impasse della ragione generata dalla

\footnotetext{
${ }^{15}$ Nonostante persistano alcune "incertezze" (MARTINELLI, 2002, p.111-112 e nota 45 a piè di pagina).
} 
coesistenza di due possibilità per sé non contraddittorie (la tesi, nel presente caso sarebbe rappresentata dall'argomento mendelssohniano, l'antitesi da quello kantiano) e forse lo scopo di Kant è proprio quello di evidenziare come l'utilizzo non fenomenico di categorie quantitative e qualitative in merito alla sostanza pensante, pur non mettendo in discussione l'esistenza di un "principio pensante", non è in grado di fornire una spiegazione che sia definitiva circa il carattere della semplicità di tale principio (nonché della sua identità, dato che anche la coscienza di sé, fondamento del concetto di Person, è passibile di remissio o illanguidimento). Mentre nella prima edizione della Kritik la situazione antinomica in cui si cadeva, in relazione all'idea psicologica, riguardava la semplicità nella misura in cui quest'ultima era funzionale alle contrapposizioni anima-corpo, inestensione-estensione, indecomponibilitàscomponibilità (le contrapposizioni che interessavano alla questione platonico-leibniziano-mendellsohniana dell'immortalità del principio spirituale pensante) e, per queste ragioni, poteva essere definita un'“antinomia estrinseca" della psicologia (carattere "ibrido" per cui sia nel 1781 che nel 1787 tali discorsi trovano trattazione nella seconda delle antinomie cosmologiche), Kant intende ora proporre un'“antinomia intrinseca”, che riguardi cioè la sola anima e le sue facoltà.

Ciò non significa che l'idea di creare un ribaltamento prospettico a favore delle istanze del materialismo venga meno nella seconda edizione della Kritik: la remissio consente infatti, agli occhi del pensatore di Königsberg, di applicare il modello atomistico alla sostanza dell'anima, che, per quando una, è pur sempre composta di gradi intensivi e quindi de-componibile e ri-componibile. In tal modo, da un unico quantum di esistenza, come un numero può essere scisso nelle unità che lo compongono, così si può ottenere una pluralità di sussistenze e, da queste, una nuova sostanza "una", con uno scambio dinamico che coinvolge tanto l'aspetto intensivo, quanto la materia. Una soluzione che, nella seconda nota alla Widerlegung, Kant afferma tuttavia di non condividere:

Ich bin weit entfernt, dergleichen Hirngespinsten den mindesten Werth oder Gültigkeit einzuräumen, auch haben die obigen Principien der Analytik hinreichend eingeschärft, von den Kategorien (als der der Substanz) keinen anderen als Erfahrungsgebrauch zu machen. Wenn aber der Rationalist aus dem bloßen Denkungsvermögen, ohne irgend eine beharrliche Anschauung, dadurch ein Gegenstand gegeben würde, ein für sich bestehendes Wesen zu machen kühn genug ist, bloß weil die Einheit der Apperception im Denken ihm keine Erklärung aus dem Zusammengesetzten erlaubt, statt daß er besser thun würde, zu gestehen, 
er wisse die Möglichkeit einer denkenden Natur nicht zu erklären, warum soll der Materialist, ob er gleich eben so wenig zum Behuf seiner Möglichkeiten Erfahrung anführen kann, nicht zu gleicher Kühnheit berechtigt sein, sich seines Grundsatzes, mit Beibehaltung der formalen Einheit des ersteren zum entgegengesetzten Gebrauche zu bedienen?. (KrV B 417-418).

Ora, tale antinomia "intrinseca" di cui si è precedentemente parlato potrebbe essere detta un'"antinomia matematica della psicologia razionale", nel senso che le due posizioni risulterebbero ugualmente false, per la pretesa di voler determinare il noumeno rimanendo sul piano del fenomeno; e tuttavia assume a mio avviso una portata differente non appena si inalvei tale discorso all'interno della seconda antinomia cosmologica, quella in merito alla divisibilità del mondo. Si porti l'attenzione alle due Anmerkungen: nell'Anmerkung zur Antithesis, Kant ricorda come l'affermazione dogmatica (mendelssohniana) circa la semplicità dell'anima ( $\mathrm{Daß}$ der Gegenstand des inneren Sinnes, das Ich, was da denkt, eine schlechthin einfache Substanz sei»: KrV A 443/B 471) vada incontro a precisi "limiti" (la semplice rappresentazione "io", senza alcuna determinazione sintetica, è semplice, mentre l'io con le sue determinazioni fornite dal senso interno è semplice come "non-composto" secondo il criterio della quantità estensiva).

Quanto all'Anmerkung zur Thesis, quest'ultima si apre con la definizione del concetto di "tutto" che costituisce il presupposto della tesi:

Wenn ich von einem Ganzen rede, welches nothwendig aus einfachen Theilen besteht, so verstehe ich darunter nur ein substantielles Ganzes, als das eigentliche Compositum, d. i. die zufällige Einheit des Mannigfaltigen, welches abgesondert (wenigstens in Gedanken) gegeben, in eine wechselseitige Verbindung gesetzt wird und dadurch Eines ausmacht. (KrV A 438/B 466).

Ciò posto, Kant sottolinea come la dimostrazione dell'esistenza del "semplice" a partire dal "composto" cessa di essere conclusiva e di avere un qualche valore nella misura in cui la si estende indifferentemente "a tutto il composto", senza cioè fare attenzione alla distinzione fenomeno-noumeno. Da una parte, infatti, come è emerso dall'Estetica trascendentale, lo spazio e il tempo non possono essere considerati dei composita; dall'altra, nemmeno i gradi del mutamento possono nemmeno essere considerati dei "composti", 
perché si tratta solo di "stati" di una sostanza per sé sussistente. Così, Kant conclude:

\begin{abstract}
Man kann also den Beweis für die Nothwendigkeit des Einfachen, als der Bestandtheile alles substantiellen Zusammengesetzten, und dadurch überhaupt seine Sache leichtlich verderben, wenn man ihn zu weit ausdehnt und ihn für alles Zusammengesetzte ohne Unterschied geltend machen will, wie es wirklich mehrmals schon geschehen ist. Ich rede übrigens hier nur von dem Einfachen, sofern es nothwendig im Zusammengesetzten gegeben ist, indem dieses darin als in seine Bestandtheile, aufgelöset werden kann. Die eigentliche Bedeutung des Wortes Mon as (nach Leibnizens Gebrauch) sollte wohl nur auf das Einfache gehen, welches un mittelbar als einfache Substanz gegeben ist (z. B. im Selbstbewußtsein) und nicht als Element des Zusammengesetzten, welches man besser den Atomus nennen könnte. (KrV A 440-442/B 468-470).
\end{abstract}

Come si evince dall'ultimo passaggio, il pensatore di Königsberg pare aprire a una soluzione noumenica in relazione alla semplicità (come accadrà nella prima delle antinomie dinamiche): "monade", leibnizianamente intesa, ossia in senso forte, può a buon diritto dirsi ciò che è dato "immediatamente" come sostanza semplice e l'autocoscienza fornisce una tale immediata semplicità. L'io dell'"io penso", non più solo rappresentazione "vuota" che accompagna ogni contenuto psichico in quanto tale e nemmeno rappresentazione del senso interno (ciò che dell'io si manifesta a se stesso), ma come "intuizione empirica indeterminata" è, esiste (la proposizione Ich existiere, osserva Kant nel 1787, è "identica" alla proposizione Ich denke: $\mathrm{KrV}$ B 422, nota a piè di pagina) e la modalità di tale esistere è la semplicità.

\title{
4 CONCLUSIONE: UNO “SQUARCIO” SULL'Io NOUMENICO
}

Lungi dall'essere una figura secondaria, Moses Mendelssohn è presente in entrambe le edizioni della Kritik: nel 1781, le tesi in merito alla semplicità della sostanza pensante esposte dal pensatore ebreo nel Phaedon (benché quest'opera non sia espressamente citata), sono al centro del secondo paralogismo; nel 1787, nonostante la rivisitazione della sezione dedicata ai Paralogismi (la presentazione degli ultimi tre paralogismi in coda al primo, a causa di quello che Faggiotto definisce "il carattere pregiudiziale del primo paralogismo": FAGGIOTTO, 1989, p.80), la "discussione” con Mendelssohn in merito alla semplicità della Seele diviene esplicita e si fa ancora più feconda 
rispetto alla prima edizione della Kritik. In ciò risiede forse la ragione dell'inserimento di una speciale sezione interamente dedicata all'argomento mendelssohniano, un'aggiunta rispetto all'edizione del 1781 che certo non era strettamente necessaria ai fini del discorso sui paralogismi e costituisce un'incongruenza rispetto all'intenzione apertamente dimostrata da Kant nel 1787 di voler compendiare in uno solo le riflessioni sui quattro paralogismi dell'anima.

Essa fornisce infatti a Kant l'occasione per istituire una specie di corrispondenza tra i paralogismi psicologici della ragion pura e le antinomie cosmologiche: lo scopo di Kant è quello di mostrare come la ragione non incorra solo nel paralogismo della sostanzialità dell'Ich denke, ma, indagando intorno alla semplicità dell'anima, cada nel secondo conflitto cosmologico riguardante la divisibilità (all'infinito o meno) della totalità degli enti (né l'anima potrebbe risultare esclusa da tale questione cosmologica essendo parte del mondo).

Diversamente da ciò che accade nelle antinomie matematiche, l'“antinomia psicologica" appare tuttavia proiettata verso l'ambito delle antinomie dinamiche: questo è quello che Kant lascia intendere sulla fine dell'Anmerkung zur Thesis della seconda antinomia cosmologica parlando della possibilità che nell'autocoscienza qualcosa sia dato "immediatamente" come "semplice".

Occorre quindi passare all'io del piano noumenico per trovare uno spiraglio circa la questione della semplicità del soggetto pensante. Con l'attenzione di distinguere in modo preciso le osservazioni che si collocano sul piano scientifico dello studio dell'io dei fenomeni del senso interno, da quelle che riconsiderano il problema nella sua globalità e si caratterizzano per una loro precisa portata metafisica, che consente di formulare in modo preciso quella che ancora Kant indicava come una "dottrina dell'anima".

POGGI, Davide. Paralogisms and antimonies: Kantian reflections on the concepts of Seele and Ich denke. Trans/Form/Ação, Marília, v. 37, p. 37-58, 2014. Edição Especial.

\footnotetext{
ABSTRACT: This essay focuses on the presence, in the section of the Dialektik dedicated to the issue of the Seele and the psychological paralogisms, of an "antinomic" approach regarding, in particular, the debate on the simplicity of the soul. In the examination of Mendelssohn's thesis concerning the soul's incorruptibility, Kant shows that it is possible to assume, together with the "extensive" criterion, an "intensive" criterion which leads to admitting the "decomposability" of the soul and the possibility of
} 
its annibilatio per remissionem. Thus, as happens in the antinomies, we are faced with two theses on the "simplicity" of the soul which are both non-contradictory, but, at the same time, opposed to each other. These reflections, proposed in the 1787 edition of the Kritik (and which seem to refer back to and refine the considerations in the 1781 edition on the simplicity of the "thinking subject" and Ich denke), play an important role and show their full value in the context of the second mathematical antinomy.

KEYWORDS: Rational psychology. Paralogisms. Ich denke. Seele. Antinomies. Extensive/intensive quantity. Simplicity of the soul.

\section{Bibliografia}

ALTMANN, Alexander. Moses Mendelssohn: a biographical study. Alabama: University of Alabama Press, 1973.

BAUMGARTEN, Alexander Gottlieb. Metaphysica. Halae Magdeburgicae: Hemmerde, $1779^{7}$.

FAGGIOTTO, Pietro. Introduzione alla metafisica kantiana dell'analogia. Milano: Massimo, 1989.

FALKENSTEIN, L. A Double Edged Sword? Kant's refutation of Mendelssohn's proof of the immortality of the soul and its implications for his theory of matter. Studies in the History and Philosophy of Science, v. 29, n.4, p. 561-588, 1998.

GUYER, P. Mendelssohn and Kant. One source of the critical philosophy. Philosophical Topics, v. 19, n. 1, p. 119-152, 1991.

KANT, Immanuel. Gesammelte Schriften. Hrsg. von der Keoniglich Preussischen Akademie der Wissenschaften. Berlin: Walter De Gruyter, 1910-1983. 28v.

KUEHN, M. David Hume and Moses Mendelssohn. Hume Studies, v. 21, n. 2, p. $197-$ 220, 1995 .

LOCKE, John. An Essay concerning human understanding. Oxford: Clarendon Press, 1975.

. Reply to the Right Reverend Bishop of Worcester's Answer to his Second Letter. In: . Works. London: Routledge-Thoemmes Press, 1997. v. 4, p. 190-499.

MARTINELLI, R. Kant, Mendelssohn e l'immortalità dell'anima. Studi Kantiani, v. 15 , p. 93-126, 2002.

MENDELSSOHN, Moses. Phaedon oder über die Unsterblichkeit der Seele in drey Gesprächen. In: . Gesammelte Schriften. Jubiläeumausgabe. Stuttgart-Bad Cannstatt: Frommann-Holzboog, 1972. v. 3, pt. 1, p. 1-128.

. Fedone: sull'immortalità dell'anima in tre dialoghi. Tr. it. a cura di Francesco Tomasoni. Brescia: Morcelliana, 2009 ${ }^{1}$. Tit. orig.: Phaedon oder über die Unsterblichkeit der Seele in drey Gesprächen. 
Morgenstunden, oder Vorlesungen über das Daseyn Gotte. In: Gesammelte Schriften. Jubiläeumausgabe. Stuttgart-Bad Cannstatt: Frommann-Holzboog, 1974. v. 3, pt. 2.

MEYER, Hermann M. Moses Mendelssohn Bibliographie: Mit einigen Ergänzungen zur Geistesgeschichte des ausgehenden 18. Jahrhunderts. Berlin: Walter de Gruyter, 1965.

PARDEY, U. Über Kants Widerlegung des Mendelssohnschen Beweises der Beharrlichkeit der Seele. Kant-Studien, v. 90, n. 3, p.257-284, 1999.

POGGI, Davide. Kant e Locke: "Tracce" lockiane nella Kritik der reinen Vernunft. Philosophical Readings, v. 2, n.1, p. 247-282, 2010. Consultabile online sul sito: www. philosophicalreadings.com

SASSEN, Brigitte. Kant and Mendelssohn on the Implications of the I Think. In: LENNON, Thomas M.; STAITON, Robert J. (Eds.). The Achilles of rationalist psychology. Dordrecht: Springer, 2008. p. 215-233.

TOMASONI, F. Mendelssohn and Kant: a singular alliance in the name of reason. History of European Ideas, v. 30, p. 267-294, 2004.

Mendelssohn's concept of the human soul in comparison with those of Georg Friedrich Meier and Kant. In: MUNK, Reinier (ed.). Moses Mendelssohn's metaphysics and aesthetics. Dordrecht: Springer, 2011. p. 131-157.

VIEILLARD-BARON, Jean-Louis. Kant critique de Mendelssohn: la psychologie rationelle. In: INTERNATIONALEN KANT-KONGRESSES, 4., 1974, Mainz. Akten des vierten... Hrsg. Von Gerhard Funke. Berlin: De Gruyter, 1974. pt. 2, p. 403-406.

WUERTH, Julian. The paralogisms of pure reason.In: GUYER, Paul (ed.). The Cambridge companion to Kant's critique of pure reason. Cambridge: Cambridge University Press, 2010. p. $210-244$. 
POGGI, D. 